\title{
Blinking Molecule Tracking
}

\author{
Andreas Karrenbauer Dominik Wöll \\ Zukunftskolleg, University of Konstanz \\ firstname. lastname@uni-konstanz.de
}

\begin{abstract}
We discuss a method for tracking individual molecules which globally optimizes the likelihood of the connections between molecule positions fast and with high reliability even for high spot densities and blinking molecules. Our method uses cost functions which can be freely chosen to combine costs for distances between spots in space and time and which can account for the reliability of positioning a molecule. To this end, we describe a top-down polyhedral approach to the problem of tracking many individual molecules. This immediately yields an effective implementation using standard linear programming solvers. Furthermore, we derive an unbiased estimator for diffusion coefficients of blinking molecules, which proves well in our experimental evaluation of our approach.
\end{abstract}

\section{Introduction}

The possibility to observe single fluorescent molecules in real-time has opened up a lot of new insights into the dynamics of systems in biology and material sciences. Single molecule microscopy (SMM) allows for the parallel observation of translational and rotational motion of many single fluorescent molecules beyond the diffraction limit provided that their concentration is reasonably low. However, tracking of single fluorescent molecules bears the challenge that the fluorescent spots are rather weak with a low signal/noise-ratio and show significant changes in signal intensity [1, 2, 3]. In the extreme case, a fluorescent molecule is dark for several recorded frames, a phenomenon which is termed blinking [4, 5].

The fact that the fluorescence signals of single molecules cannot be classified according to their intensity or shape in different frames and even disappear in some frames causes severe problems for single molecule tracking. Thus, many tracking algorithms which have been developed for single particle tracking (e.g. for cells) in video microscopy fail for tracking single molecules.

The way from the recorded single molecule microscopy movies to the results about their motion includes typically the following steps:

(i) determination of the positions of each fluorescent molecule,

(ii) connecting the positions to single molecule tracks, and

(iii) statistical analysis of these tracks. 


\subsection{Previous Work}

Nowadays, single molecule positions are most often determined using center of mass or Gaussian fits, with the latter being the best option for low signal-to-noise ratios of around four [6]. Usually the images are preprocessed by various filters, e.g. Mexican hat [7], before the actual localization.

After localization, the positions of subsequent frames have to be connected to tracks [8]. Different approaches have been developed for this purpose, but up to now it remains challenging to improve and develop algorithms not only for special tasks but for a universal set of problems [9].

Single Particle Tracking procedures started with the connection of one point with its closest neighbor in consecutive frames [10]. In 1999, Chetverikov et al. published a new algorithm called IPAN Tracker. 11] Using a competitive linking process that develops as the trajectories grow, this algorithm deals better with incomplete trajectories, high spot densities, faster moving particles and appearing and disappearing spots. Sbalzarini et al. used the same approach as the IPAN tracker, but did not make any assumptions about the smoothness of trajectories [12]. Their algorithm was implemented as ParticleTracker in ImageJ.

The SpotTracker [7] is a very powerful tool to follow single spots throughout one movie, but it can only proceed spot by spot. The algorithm proposed by Bonneau et al. [13] falls in the same category of greedy algorithms that iteratively compute shortest paths in space-time, which are not revised subsequently.

One of the most accurate solutions to single particle tracking is provided by multiplehypothesis tracking (MHT). This method chooses the largest non-conflicting ensemble of single particle paths simultaneously accounting for all position in each frame. Jaqaman et al. used such an approach where they first linked positions in consecutive frames by solving bipartite matching problems and combined these links into entire trajectories [14] with a post-processing step to account for missing points in a frame. Both steps were optimized independently which yielded a very likely solution of the tracking problem. Dynamic multipletarget tracing was used by Sergé et al. to generate dynamic maps of tracked molecules at high density. [15] Subtracting detected peaks from the images allows them for a detection of low intensity peaks which would be otherwise hidden in movies of high particle density. Peak positions were connected using statistical information from past trajectories.

Moreover, manual or semi-automated approaches, in which only unambiguous choices are performed automatically, are still used though there are cumbersome due to many user interaction at high particle densities.

For the analysis of the tracks, different approaches have been developed [16, 9]. The most common approach is analysis of the mean square displacement for different time intervals [17, 18, 19, 20] which can readily distinguish between different modes of motion such as normal diffusion, anomalous diffusion, confined diffusion, drift and active transport [21]. Alternatively, probability distribution of squared displacements [22] and radii of gyration [23, 24, 16] can be used to analyze single molecule tracks.

\subsection{Our Contribution}

We present a method for single molecule tracking which globally optimizes the likelihood of the connections between molecule positions fast and with high reliability even for high spot densities. Our method uses cost functions which can be freely chosen to combine costs 
for distances between spots in space and time and which can account for the reliability of positioning a molecule. Using a suitable positioning procedure, reliable tracking can be performed even for highly mobile, frequently blinking and low intensity fluorescent molecules, cases for which most other tracking algorithms fail.

In the following, we present a top-down approach for modeling molecule tracking. We unify the previous approaches in one framework. By developing a suitable polyhedral model, we show theoretically that it remains computationally tractable. A major advantage of our method is that we immediately obtain an effective software solution using standard linear programming software. Moreover, we experimentally evaluate our implementation. To this end, we use real-world data and realistic data, i.e. randomly generated according to a physical model. We qualitatively compare our tracking on the real-world data to tracks obtained by a human expert, whereas we exploit the knowledge about the ground-truth in the realistic data to quantitatively measure the impact of noise and the validity of the parameters that we have chosen. We evaluated our approach for two-dimensional tracking, but the extension to $3 \mathrm{D}$ is straight-forward. We provide our software as open source code for MATLAB using CPLEX as LP-solver (see Appendix).

\section{A Polyhedral Model for Molecule Tracking}

It is easy to see that the number of possible trajectories grows exponentially with the number of points. To tackle this combinatorial explosion [13, we consider the a top-down polyhedral approach for a concise representation in this paper. Suppose we are given a set of points $V=\left\{v_{1}, \ldots, v_{n}\right\}$. Each point has one temporal and $d$ spatial coordinates, say $\left(t_{i}, x_{i}, y_{i}\right)$, $i \in V$, with $d=2$ as used in the following for the sake of presentation. Before we further dwell on formalism, we first formulate the following conditions for a track:

- Each point has at most one predecessor.

- Each point has at most one successor.

We model the predecessor/successor relation of two points by ordered pairs. To this end, let $V_{t<t_{j}}:=\left\{v_{i} \in V: t_{i}<t_{j}\right\}$ and $A:=\left\{\left(v_{i}, v_{j}\right): v_{i} \in V_{t<t_{i}}, v_{j} \in V\right\}$. We denote the predecessor/successor relation by $f: A \rightarrow\{0,1\}$. Moreover, let $g, h: V \rightarrow\{0,1\}$ denote missing predecessors and successors, respectively. That is, $g(v)=1$ iff $v \in V$ does not have a predecessor, and $h(v)=1$ iff it does not have a successor. Let $\delta^{\text {in }}(v), \delta^{o u t}(v) \subseteq A$ denote the sets of possible predecessor/successor relations for point $v$.

Definition 1. A track partition or tracking of $V$ is a collection of disjoint tracks covering $V$, i.e. each point appears in exactly one track, which might consist of a single point.

The characteristic vector $(f, g, h)$ of a track partition is a $\{0,1\}$-vector in which the first $|A|$ entries corresponding to $f$ denote the predecessors/successor relation, the following $|V|$ entries corresponding to $g$ determine the starting points of the tracks, and the last $|V|$ entries corresponding to $h$ define the endpoints of the tracks.

Theorem 2. The tracking polytope, i.e. the convex hull of all track partitions, is described by

$$
P:=\left\{(f, g, h) \in[0,1]^{|A|+2|V|}: \forall v \in V: g(v)+f\left(\delta^{\text {in }}(v)\right)=1, h(v)+f\left(\delta^{\text {out }}(v)\right)=1\right\} .
$$


Proof. It is easy to see that the characteristic vector of a tracking is contained in $P$. Moreover, each $\{0,1\}$-vector in $P$ corresponds to a tracking. Hence, it remains to show that these are the only vertices of $P$. To this end, we prove that the constraint matrix $M$ that defines $P$ in the form $P=\{x: M x=1, x \geq 0\}$ is totally unimodular. First, we observe that $M=\left(M^{\prime} I\right)$ where $M^{\prime}$ corresponds to the $f$-variables. Hence, it suffices to show total unimodularity for $M^{\prime}$. To this end, we consider an auxiliary graph $G^{\prime}=\left(V_{1} \dot{\cup} V_{2}, E^{\prime}\right)$ at which each of $V_{1,2}$ contains a copy of each point in $V$ and $E^{\prime}$ is the set of edges that mimics the set $A$ on $V_{1} \times V_{2}$. Note that by this definition $G^{\prime}$ is bipartite. Moreover, its adjacency matrix is given by $M^{\prime}$. Hence, $M^{\prime}$ and $M$ are totally unimodular.

\subsection{Optimization}

Based on the compact representation of all possible tracks as described before, we now consider the problem of selecting an appropriate tracking out of all these possibilities. To this end, we leverage the fundamental paradigm of normal diffusion: Tracks are Markov chains, i.e. the transition probability from one state to another does only depend on the current state and not on the history that led to it. Thus, all transitions are independent random events.

Suppose that we are given the probability distributions $p_{1}: A \rightarrow[0,1]$ for the transitions and $p_{2,3}: V \rightarrow[0,1]$ denoting the probability that a point is the beginning or the end of a track, respectively. We wish to find a tracking with maximum likelihood. That is, a tracking that maximizes the joint probability of the independent random events

$$
\mathcal{L}(f, g, h)=\prod_{\substack{a \in A \\ f(a)=1}} p_{1}(a) \prod_{\substack{v \in V \\ g(v)=1}} p_{2}(v) \prod_{\substack{v \in V \\ h(v)=1}} p_{3}(v)
$$

We note that our approach is universal w.r.t. to the chosen probability distributions. For the sake of presentation, we consider a normal diffusion model in this paper. That is, the probability that after time $t$ a particle is translated by a vector $r=\left(r_{x}, r_{y}\right)$ is proportional to

$$
\exp \left(-\frac{r_{x}^{2}+r_{y}^{2}}{4 D t}\right)
$$

where $D \in \mathbb{R}$ is called diffusion coefficient.

The joint probability distribution that $N$ particles have moved by $r(1), \ldots, r(N) \in \mathbb{R}^{2}$ after one time-step of length $t$ is hence given by the product

$$
\prod_{i=1}^{N} \exp \left(-\frac{r_{x}^{2}(i)+r_{y}^{2}(i)}{4 D t}\right)=\exp \left(-\sum_{i=1}^{N} \frac{r_{x}^{2}(i)+r_{y}^{2}(i)}{4 D t}\right)
$$

Plugging this into the joint probability distribution, we obtain

$$
\mathcal{L}(f, g, h)=\exp \left[-\sum_{a \in A} \frac{r_{x}^{2}(a)+r_{y}^{2}(a)}{4 D t} \cdot f(a)+\sum_{v \in V} \ln \left(p_{2}(v)\right) \cdot g(v)+\sum_{v \in V} \ln \left(p_{3}(v)\right) \cdot h(v)\right]
$$

Due to monotonicity of the exponential function, finding the most likely tracking amounts to solve the linear programming problem

$$
\min \left\{\sum_{a \in A} c_{1}(a) f(a)-\sum_{v \in V} c_{2}(v) g(v)-\sum_{v \in V} c_{3}(v) h(v):(f, g, h) \in P\right\}
$$


Note that this formulation is again general enough to capture arbitrary separable likelihood functions $\mathcal{L}(f, g, h)$ and it is not restricted to normal diffusion.

Lemma 2.1. For all optimum solutions $(f, g, h) \in P$ and $a=(v, w) \in A$, we have

$$
f(a)=1 \quad \Rightarrow \quad c_{1}(a) \leq c_{2}(v)+c_{3}(w) .
$$

Proof. By contradiction: We would obtain a better feasible solution by setting $f(a)=0$ and $g(v)=h(w)=1$.

Although it is possible to consider different probabilities for appearing and vanishing particles, we choose a constant one, i.e. let $c_{2}(v)=c_{3}(v)=C$ for all $v \in V$. Hence, $c(a) \leq 2 \cdot C$ for all $a \in A$ which appear in any optimum solution. This inspires the definition of a tracking radius $R$ such that we will only consider predecessors and successors within that range. This dramatically limits the size of an instance and enables us to use space partition techniques to efficiently construct the tracking LP. Put differently, the restriction to a certain tracking radius for efficiency reason is justified by Lem. 2.1. In the experiments section, we will discuss suitable choices for $R$. Similarly, it makes sense to limit the number of frames that a molecule might be invisible.

\subsection{Dealing with Noise}

Since the points are usually detected from noisy images, there are false-positive and falsenegatives. That is, a spot $v \in V$ is a false-positive, if it does not correspond to any track. A false-negative is a point that does not have a correspondent in $V$.

Moreover, there might be $v, w \in V$ that correspond to the same point. To deal with these duplicates, we introduce so-called joins into $A$. That is, we allow that two points from the same frame appear in one track. We thereby maintain the integrality of our polyhedron. However, we treat these joins differently w.r.t. the objective function to reflect the special situation. We propose to set the cost of such a link to the ordinary cost of that connection plus the mean of the penalties for not having a successor and a predecessor, respectively. Taking the penalty into account is necessary to avoid 2-cycles.

Since we can only deal with points that are present in $V$, we shall avoid false-negatives in the point detection. However, a low false-negative rate often leads to a high false-positive rate. Therefore, we utilize the possibility to consider a quality measure of each detected point. That is, we reduce the cost of not tracking a point according to its quality. This can be modeled easily by multiplying $c_{2,3}(v)$ by some quality factor $q(v)$, e.g. proportional to the strength of the signal of this spot.

\section{Determination of diffusion coefficients from track positions}

A popular method to determine the diffusion coefficients from single molecule tracks is called track radius analysis (also known as radius of gyration analysis) [16, 24, 23, which uses the eigenvalues of the sample covariance matrix $Q$. The advantage of this method lies in the high accuracy of the obtained diffusion coefficients which are robust even for small trajectories. Whereas previously the relation was only verified by Monte Carlo random walk simulations of normal diffusion [25], we now give a theoretical explanation, which is in agreement with the experimental findings. 
We first observe that the original definition of the track radius $R_{T}$ as the sum of the eigenvalues of $Q$ is equivalent to the trace of $Q$, i.e. $R_{T}^{2}:=\sum_{k=1}^{d} \lambda_{k}(Q)=\operatorname{tr}(Q)$. Hence, the track radius is only determined by the variances of the individual coordinates and it is independent of the covariances between the coordinates. As mentioned before, $R_{T}$ is a biased estimator for the diffusion coefficient $D$. That is, under certain fixed conditions, e.g. no blinking, fixed track lengths, the diffusion coefficient is proportional to $R_{T}$.

In the following, we show how to obtain an unbiased estimator for the diffusion coefficient in a normal diffusion model, which also accounts for blinking and variable track lengths. That is, we consider position updates of the form

$$
x_{i}=x_{i-1}+s_{i} \cdot n_{i} \quad \text { for } i>1,
$$

where $n_{i}$ is a standard normal random variable (i.e. $\mu=0, \sigma=1$ ) and the step-lengths $s_{i}$ are dependent on the diffusion coefficient and the time between the observations. Hence, the coordinates $x_{i}$ are Gaussian random variables with

$$
\begin{aligned}
E\left[x_{i}\right] & =x_{1}+\sum_{k=2}^{i} s_{k} E\left[n_{k}\right]=x_{1} \\
E\left[x_{i} x_{j}\right] & =x_{1}^{2}+x_{1} \sum_{k=2}^{i} s_{k} E\left[n_{k}\right]+x_{1} \sum_{k=2}^{j} s_{k} E\left[n_{k}\right]+\sum_{k=2}^{i} \sum_{\ell=2}^{j} s_{k} s_{\ell} E\left[n_{k} n_{\ell}\right]=x_{1}^{2}+\sum_{k=2}^{\min \{i, j\}} s_{k}^{2}
\end{aligned}
$$

In the case of normal diffusion, we have $s_{k}^{2}=2 D\left(t_{k}-t_{k-1}\right)$, where $t_{k}$ denotes the time of the $k$ th observation. Thus, we may write

$$
E\left[x_{i} x_{j}\right]=x_{1}^{2}+2 D\left(t_{\min \{i, j\}}-t_{1}\right) .
$$

We use the unbiased estimator for the variance of the $x$-coordinates of a track $x_{1}, \ldots, x_{N}$, which is determined as

$$
\hat{\sigma}_{x}^{2}:=\frac{1}{N-1} \sum_{i=1}^{N}\left(x_{i}-\frac{1}{N} \sum_{k=1}^{N} x_{k}\right)^{2}=\frac{1}{N-1} \sum_{i=1}^{N} x_{i}^{2}-\frac{1}{N(N-1)} \sum_{i=1}^{N} \sum_{j=1}^{N} x_{i} x_{j}
$$

In expectation, we get

$$
\begin{aligned}
(N-1) \cdot E\left[\hat{\sigma}_{x}^{2}\right] & =\sum_{i=1}^{N} E\left[x_{i}^{2}\right]-\frac{1}{N} \sum_{i=1}^{N} \sum_{j=1}^{N} E\left[x_{i} x_{j}\right] \\
& =N x_{1}^{2}+2 D \sum_{i=1}^{N}\left(t_{i}-t_{1}\right)-N x_{1}^{2}-\frac{2 D}{N} \sum_{i=1}^{N} \sum_{j=1}^{N}\left(t_{\min \{i, j\}}-t_{1}\right) \\
& =\frac{2 D}{N} \sum_{i=2}^{N}(2 i-1-N)\left(t_{i}-t_{1}\right) .
\end{aligned}
$$

Because the random displacements of the $\mathrm{x}$ - and $\mathrm{y}$-coordinates are independent identically distributed, we obtain the same for $E\left[\hat{\sigma}_{y}\right]$. This motivates the definition of

$$
\hat{D}:=\frac{N(N-1)}{4} \cdot \frac{\hat{\sigma}_{x}^{2}+\hat{\sigma}_{y}^{2}}{\sum_{i=2}^{N}(2 i-1-N)\left(t_{i}-t_{1}\right)}=\frac{N(N-1)}{4 \sum_{i=2}^{N}(2 i-1-N)\left(t_{i}-t_{1}\right)} \cdot R_{T}^{2},
$$


which is an unbiased estimator for the diffusion coefficient in $d$ dimensions because $E[\hat{D}]=D$. For the special case of uniform time-steps, we substitute $t_{i}=i \cdot \tau$ and obtain

$$
\hat{D}:=\frac{3}{2 \tau} \cdot \frac{\hat{\sigma}_{x}^{2}+\hat{\sigma}_{y}^{2}}{N+1}=\frac{3}{2(N+1) \tau} \cdot R_{T}^{2} .
$$

Note the we defined $\hat{\sigma}$ and $R_{T}$ as the unbiased estimators of the variance and via the sample covariance matrix, respectively. If the maximum likelihood estimators, i.e. normalization by $1 / N$ instead of $1 /(N-1)$, are used, then this is simply accounted by an addition factor of $N /(N-1)$.

Since the localization of the centers of the spots is subject to some uncertainty, we extend our model as follows. We define $\tilde{x}_{i}:=x_{i}+\varepsilon_{i}$, where $\varepsilon_{i} \sim \mathcal{N}\left(0, \varepsilon^{2}\right)$ are independent normally distributed random variables with variance $\varepsilon^{2}$. Hence, we obtain

$$
\begin{aligned}
E\left[\tilde{x}_{i}\right] & =E\left[x_{1}\right] \\
E\left[\tilde{x}_{i} \tilde{x}_{j}\right] & =E\left[x_{i} x_{j}\right]+E\left[\varepsilon_{i}\right] \cdot E\left[x_{j}\right]+E\left[x_{i}\right] \cdot E\left[\varepsilon_{j}\right]+E\left[\varepsilon_{i} \varepsilon_{j}\right]=E\left[x_{i} x_{j}\right]+\varepsilon^{2} \delta_{i j}
\end{aligned}
$$

with Kronecker's $\delta_{i i}=1$ and $\delta_{i j}=0$ for $i \neq j$. Since we can estimate the track variances only based on the measured data $\tilde{x}_{i}$, we get in expectation

$$
\begin{aligned}
(N-1) \cdot E\left[\hat{\sigma}_{x}^{2}\right] & =\sum_{i=1}^{N} E\left[\tilde{x}_{i}^{2}\right]-\frac{1}{N} \sum_{i=1}^{N} \sum_{j=1}^{N} E\left[\tilde{x}_{i} \tilde{x}_{j}\right] \\
& =\sum_{i=1}^{N} E\left[x_{i}^{2}\right]+N \varepsilon^{2}-\frac{1}{N} \sum_{i=1}^{N} \sum_{j=1}^{N} E\left[x_{i} x_{j}\right]-\varepsilon^{2} \\
& =(N-1) \varepsilon^{2}+\frac{2 D}{N} \sum_{i=2}^{N}(2 i-1-N)\left(t_{i}-t_{1}\right) .
\end{aligned}
$$

Hence, we now have a bias in the estimator $\hat{D}$ as defined above depending on $\varepsilon$ :

$$
E[\hat{D}]=D+\frac{N(N-1) \varepsilon^{2}}{2 \sum_{i=2}^{N}(2 i-1-N)\left(t_{i}-t_{1}\right)},
$$

Thus, uniform time-steps yield

$$
E[\hat{D}]=D+\frac{3 \varepsilon^{2}}{(N+1) \tau} \stackrel{N \rightarrow \infty}{\longrightarrow} D .
$$

Put differently, the longer the tracks the less influential are the localization errors. Note that this derivation also holds for any independent identically distributed localization errors with mean 0 and variance $\varepsilon^{2}$.

\section{Experiments}

So far, we described a generic approach for tracking blinking molecules. In this section, we propose our choice for the cost-function, i.e. $c\left(v_{i}, v_{j}\right)=\left(x_{i}-x_{j}\right)^{2}+\left(y_{i}-y_{j}\right)^{2}+\left(t_{i}-t_{j}\right)^{2}$, which is validated experimentally. 

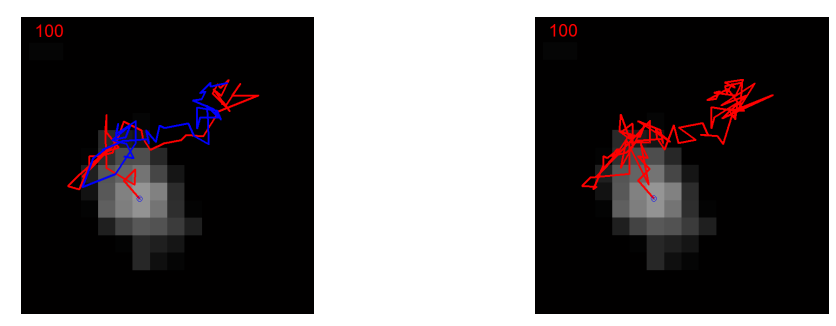

Figure 1: Tracking with $\Delta x^{2}+\Delta y^{2}$ on the left and with $\Delta x^{2}+\Delta y^{2}+\Delta t^{2}$ on the right. Two interleaved tracks are produced for one molecule on the left, whereas there is exactly one on the right.

The rationale for using this function is based on the following observation: if time is not penalized, then track fragmentation becomes more likely as shown in Fig. 1 .

Thus, we introduced the superlinear term $\Delta t^{2}$ such that two time steps of length 1 are cheaper than one time step of length 2 . Hence, the spatial distance is mainly responsible for comparing positions within the same frame. We consider closer destinations to be more likely. Therefore, we do not use the time in this part of the objective.

In this section, we also briefly discuss the dependencies of the results on parameters such as the tracking radius $R$ (a more in-depth analysis will be published elsewhere [26]). To this end, we first consider a controlled testing environment based on the normal diffusion model mentioned above. We thereby obtain realistic randomly generated instances. Though the experiments with the simulated realistic data has the advantage that we know the ground truth and thus we can quantify the deviation of the computed results in certain situations, we shall also validate our approach on real-world instances. To this end, we compare the diffusion coefficients obtained manually by a human expert with our automated approach in the final subsection of this paper.

\subsection{Realistic Data}
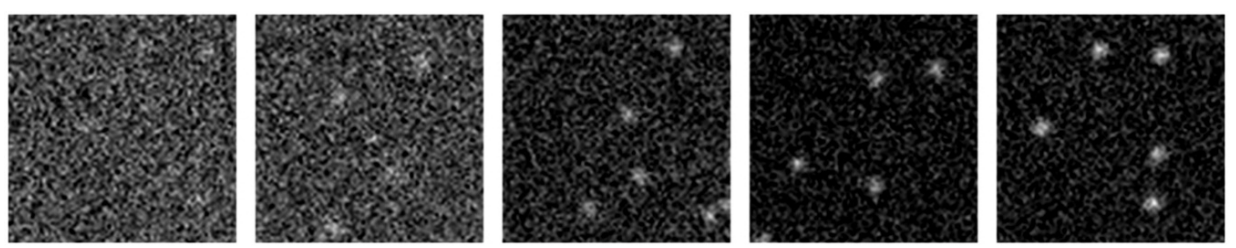

Figure 2: Different signal-to-noise ratios $(1,2,3,4$, and 5 from left to right).

Synthetic trajectories were generated to test our localization and tracking algorithms. Random walk simulations with 500 steps were performed using four different diffusion coefficients $\left(10^{-15}, 10^{-14}, 10^{-13}\right.$, and $\left.10^{-12} \mathrm{~m}^{2} \mathrm{~s}^{-1}\right)$, five different signal-to-noise ratios $(1,2,3,4$, and 5, see Fig. 2) and five different particle densities (100, 200, 300, 400 and 500 particles per frame). These values can be found in many tracking challenges of practical importance, but were also chosen to determine the sensitivity of our algorithms. The initial $\mathrm{x}$ - and $\mathrm{y}$-position of each spot was chosen uniformly at random in the range [0;500]. The single molecule trajectories were used to construct movies with 500 frames. Two dimensional Gaussian functions were constructed with their center located at the positions obtained from the random walk 
simulations and their widths being diffraction limited (ca. $300 \mathrm{~nm}$ ). The time between consecutive frames was chosen as $0.1 \mathrm{~s}$ and the resolution as $100 \mathrm{~nm}$ per pixel. Gaussian white noise was added to the movies corresponding to the signal-to-noise ratio defined as with the amplitude of the center of the 2D Gaussian and the standard deviation of the Gaussian white noise.

\subsubsection{Determination of tracking accuracy for simulated data}

Tracking procedures have to meet several conditions to be suitable. Apart from practical aspects such as tracking speed and memory consumption, the number of false-positives is the key factor which has to be minimized in order to obtain reliable results. Similarly to the definition of false-positive and false-negative w.r.t. particle locations, false positives in this context are connections between positions in different frames which have been set even though the positions do not belong to the same molecule/particle. They can result in severe errors in single molecule tracking and cause wrong interpretations of collected data. Thus, the number of false positives should be kept as low as possible. False negatives are connections between positions in different frames which are not recognized by the tracking algorithm.

The fraction of false positives and false negatives for movies of different diffusion coefficients and signal-to-noise-ratios was calculated comparing ground truth and analyzed connections between points. The fraction of false positive connections decreases from $13 \%$ to $3 \%$ as the $\mathrm{S} / \mathrm{N}$-ratio increases from 1 to 5 . False negatives particularly occur with fast moving molecules if the tracking radius is not chosen carefully. The reason is as follows. Since the distribution of displacements can be expressed [27] as

$$
p(r, t)=\frac{r}{2 D t} \exp \left(-\frac{r^{2}}{4 D t}\right),
$$

the probability of finding the destination within a radius of $\mathrm{R}$ is

$$
P(R, t)=\int_{0}^{R} p(r, t) d r=1-\exp \left(-\frac{R^{2}}{4 D t}\right) .
$$

Thus, picking the tracking radius too low yields biased false-negatives and hence an underestimation of the diffusion coefficients. However, the tempting choice of an excessive tracking radius does not only require much more computational resources, but may also lead to an overestimation of the diffusion coefficients if there occur leaps in the tracks due to false-positives (in particular with high particle densities). We propose to choose the tracking radius such that a displacement is smaller with a probability of about $99 \%$. Since those leaps are easily determined in a post-processing steps, a repetition of the tracking with different radii in a feedback loop is feasible.

Positioning and tracking are the key steps in the determination of diffusion coefficients. To distinguish the errors appearing in these steps, we analyzed four different cases shown in Fig. 3 .

In case (1), the distribution of diffusion coefficient was directly calculated from the ground truth tracks. Though all tracks were created with respect to fixed diffusion coefficients, the calculation yields peaked distributions around the true values because of the finite number of sample points in each track.

In a second set of analysis (case 2) the same ground truth positions were tracked using our polyhedral model solved with CPLEX. Good results were obtained except for fast molecules 


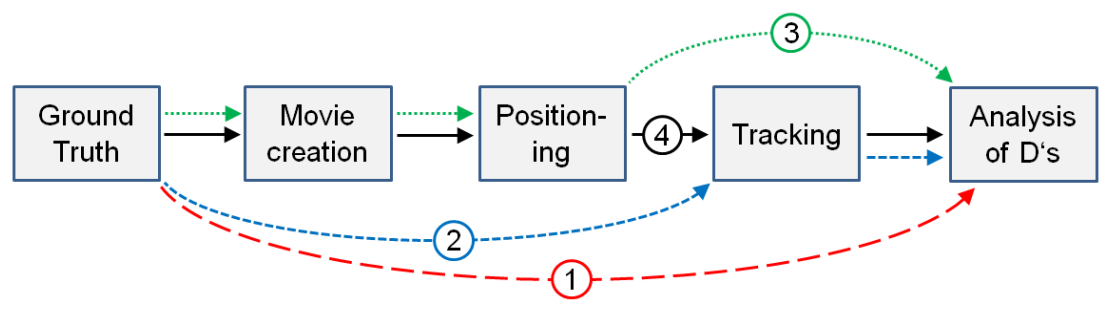

Figure 3: Steps taken from the simulated ground truth data to the analysis of the trajectories. For analysis of the accuracy of each single step different paths were considered which are labeled 1 to 4 .

and high densities. That is, the probability of foreign spots moving into the tracking range of another molecule is too high, and thus the tracking algorithm in general returns diffusion coefficients lower than the real value.

The third set of analysis, case 3, allows for an investigation of the influence of positioning inaccuracies on the distribution of diffusion coefficients. Movies were constructed from the ground truth positions with different $\mathrm{S} / \mathrm{N}$-ratios. Our positioning algorithm was applied to these movies and, where possible, the positions matched to the positions of the ground truth tracks. With the determined positions of each track, a diffusion coefficient was obtained. The distribution of diffusion coefficients of these tracks resembles the distributions of the ground truth tracks with the exception of low diffusion coefficients with low S/N-ratios where the poor localization accuracy results in a seemingly higher diffusion coefficient than simulated.

Case 4 describes the procedure which is applied for real movies to determine single molecule diffusion coefficients. Spots in movies are positioned, the positions tracked and a diffusion coefficient calculated from these tracks. For high S/N-ratios the obtained distributions are similar to the simulated distributions. Two trends can be observed in particular at low $\mathrm{S} / \mathrm{N}$ - ratios:

(i) molecules with low diffusion coefficients tend to be analyzed as being faster as they were simulated, and

(ii) analysis of the motion of fast molecules in average results in a lower diffusion coefficient as the ground truth data.

The former observation can be explained by the poorer localization accuracy at low S/N-ratios. This inaccuracy resembles diffusion and thus a low diffusion coefficient will be assigned even to immobile molecules. The localization accuracy determines the lowest diffusion coefficient which can be determined by the corresponding experimental settings.

\subsubsection{Tracking of blinking data}

In the previous subsection, points were missing due to false negatives in the localization at low S/N-ratios. However, even with perfect localization missing points may occur naturally in real-world experiments because the fluorescence intensity of single molecules is typically not constant, but shows blinking behavior due to photochemical or photophysical quenching processes [1] such as intersystem crossing to the triplet state [28, 29, 30] or electron transfer [31]. The lengths of on- and off-times typically show a power law distribution [1]. In order to simulate blinking behavior, we generated on- and off-times for our simulated tracks using the 
following procedure. At the beginning a molecule was u.a.r. set as on or off. The number of frames remaining in this state was determined randomly from a probability distribution with $P(t) \sim(t / \tau)^{\alpha}$. We chose realistic values for $\tau$ and $\alpha$, i.e. $\tau=1$ s and $\alpha=-2$, respectively.
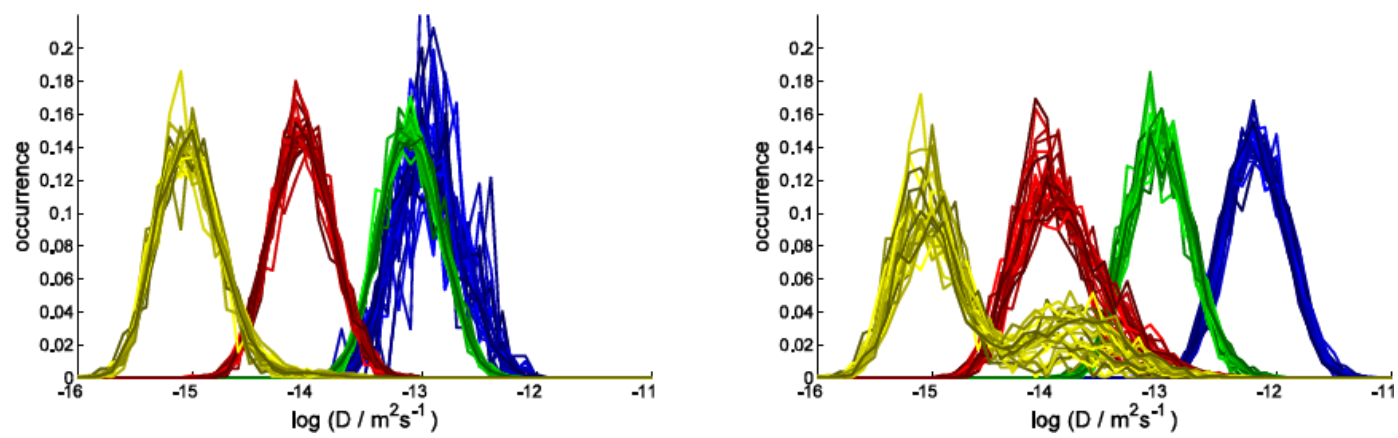

Figure 4: Distributions of diffusion coefficients obtained after tracking of blinking data for a maximum step length of 5 (left) and 15 (right).

The analyzed distributions of diffusion coefficients for tracking of blinking ground truth data are shown in Fig. 4. For a maximum step length of 5 pixels, the distributions of diffusion coefficients resemble the ground truth data except for faster molecules with diffusion coefficients above $10^{-13} \mathrm{~m}^{2} \mathrm{~s}^{-1}$ where a step length of 5 pixels is not sufficient for tracking as discussed before. Tracking with a maximum step length of 15 pixels gives good results for fast diffusing molecules, but for simulated diffusion coefficients of $10^{-14} \mathrm{~m}^{2} \mathrm{~s}^{-1}$ and below results a long tail or even a second band at higher $\mathrm{D}$ values (see Fig. 4 (right)). The deviation of distribution from the ground truth distribution is caused by tracks which include at least one large jump from one molecule to another one which results in a significant increase of the track radius.

\subsection{Real-World Data}

The real-world data were obtained from single molecule fluorescence widefield experiments during the bulk radical polymerization of styrene to polystyrene. The motion of single perylene diimide fluorophores was observed at various monomer-to-polymer-conversions and thus different viscosities which allowed us to probe a broad range of diffusion coefficients. The interested reader is referred to [25].

Before this project, the tracks were constructed semi-manually due to the lack of a satisfying alternative. That is, only a simple search in the neighborhood of the points was performed automatically as long as there were no ambiguities, i.e. only one localized point within the tracking radius and no competition among potential predecessors. In the case when the automatic continuation of the tracks fails, the user was presented with 10 consecutive frames of the movie with the options to select a successor among the alternative, to introduce a new spot that was not detected by the localization, or to end the track. Needless to say that this was a tedious task, which took several working days to complete the tracking of a 5-minute-movie with high particle density. The advantage of this method is that the human expert maintains the full control over the process and the pattern recognition capabilities of the human brain is leveraged to resolve situations in which the image processing tools fail. On the other hand, these possibilities are also a disadvantage as the user might introduce systematic errors in the data and it is unlikely that a repetition of the task yields exactly the same results. 
It remains to show that our automatic method not only works for realistic data but also in the real-world. To this end, we compare the average diffusion coefficients obtained from 6 movies by manual and automatic tracking:

\begin{tabular}{|l|llllll|l|}
\hline manual & 0.019 & 0.053 & 0.126 & 0.537 & 1.166 & 4.864 & \multirow{2}{*}{$\cdot 10^{-13} \mathrm{~m}^{2} \mathrm{~s}^{-1}$} \\
automatic & 0.023 & 0.054 & 0.132 & 0.509 & 1.054 & 4.372 & \\
\hline
\end{tabular}

\section{References}

[1] F. Cichos, C. von Borczyskowski, and M. Orrit, "Power-law intermittency of single emitters," Current Opinion in Colloid ES Interface Science, vol. 12, no. 6, pp. 272-284, 2007.

[2] M. Lippitz, F. Kulzer, and M. Orrit, "Statistical evaluation of single nano-object fluorescence," Chemphyschem, vol. 6, no. 5, pp. 770-789, 2005.

[3] D. Bingemann, “Analysis of 'blinking' or 'hopping' single molecule signals with a limited number of transitions," Chemical Physics Letters, vol. 433, no. 1-3, pp. 234-238, 2006.

[4] W. Göhde, U. C. Fischer, H. Fuchs, J. Tittel, T. Basché, C. Bräuchle, A. Herrmann, and K. Müllen, "Fluorescence blinking and photobleaching of single terrylenediimide molecules studied with a confocal microscope," Journal of Physical Chemistry A, vol. 102, no. 46, pp. 9109-9116, 1998. Times Cited: 20 Gohde, W Fischer, UC Fuchs, H Tittel, J Basche, T Brauchle, C Herrmann, A Mullen, K.

[5] W. T. Yip, D. H. Hu, J. Yu, D. A. Vanden Bout, and P. F. Barbara, "Classifying the photophysical dynamics of single- and multiple-chromophoric molecules by single molecule spectroscopy," Journal of Physical Chemistry A, vol. 102, no. 39, pp. 75647575, 1998. Times Cited: 193 Yip, WT Hu, DH Yu, J Vanden Bout, DA Barbara, PF.

[6] M. K. Cheezum, W. F. Walker, and W. H. Guilford, "Quantitative comparison of algorithms for tracking single fluorescent particles," Biophysical Journal, vol. 81, no. 4, pp. 2378-2388, 2001.

[7] D. Sage, F. R. Neumann, F. Hediger, S. M. Gasser, and M. Unser, "Automatic tracking of individual fluorescence particles: Application to the study of chromosome dynamics," Ieee Transactions on Image Processing, vol. 14, no. 9, pp. 1372-1383, 2005. ISI Document Delivery No.: 956SF Times Cited: 93 Cited Reference Count: 28 Sage, D Neumann, FR Hediger, F Gasser, SM Unser, M Ieee-inst electrical electronics engineers inc Piscataway.

[8] T. Schmidt, G. J. Schutz, W. Baumgartner, H. J. Gruber, and H. Schindler, "Imaging of single molecule diffusion," Proceedings of the National Academy of Sciences of the United States of America, vol. 93, no. 7, pp. 2926-2929, 1996. ISI Document Delivery No.: UD375 Times Cited: 319 Cited Reference Count: 30 Natl acad sciences Washington.

[9] M. J. Saxton, "Single-particle tracking: conneting the dots," Nature Methods, vol. 5, pp. $671-672,2008$.

[10] R. N. Ghosh and W. W. Webb, "Automated detection and tracking of individual and clustered cell-surface low-density-lipoprotein receptor molecules," Biophysical Journal, 
vol. 66, no. 5, pp. 1301-1318, 1994. ISI Document Delivery No.: NG923 Times Cited: 153 Cited Reference Count: 45 Ghosh, rn webb, ww Biophysical society Bethesda.

[11] D. Chetverikov and J. Verestoy, "Feature point tracking for incomplete trajectories," Computing, vol. 62, no. 4, pp. 321-338, 1999. ISI Document Delivery No.: 212MN Times Cited: 29 Cited Reference Count: 10 Chetverikov, D Verestoy, J Springer-verlag wien Vienna.

[12] I. F. Sbalzarini and P. Koumoutsakos, "Feature point tracking and trajectory analysis for video imaging in cell biology," Journal of Structural Biology, vol. 151, no. 2, pp. 182-195, 2005. ISI Document Delivery No.: 954XA Times Cited: 133 Cited Reference Count: 31 Sbalzarini, IF Koumoutsakos, P Academic press inc elsevier science San diego.

[13] S. Bonneau, M. Dahan, and L. D. Cohen, "Single quantum dot tracking based on perceptual grouping using minimal paths in a spatiotemporal volume," Ieee Transactions on Image Processing, vol. 14, no. 9, pp. 1384-1395, 2005. Times Cited: 54 Bonneau, S Dahan, M Cohen, LD.

[14] K. Jaqaman, D. Loerke, M. Mettlen, H. Kuwata, S. Grinstein, S. L. Schmid, and G. Danuser, "Robust single-particle tracking in live-cell time-lapse sequences," Nature Methods, vol. 5, no. 4, pp. 695-702, 2008.

[15] A. Sergé, N. Bertaux, H. Rigneault, and D. Marguet, "Dynamic multiple-target tracing to probe spatiotemporal cartography of cell membranes," Nature Methods, vol. 5, pp. 687$694,2008$.

[16] L. C. C. Elliott, M. Barhoum, J. M. Harris, and P. W. Bohn, "Trajectory analysis of single molecules exhibiting non-brownian motion," Physical Chemistry Chemical Physics, vol. 13, pp. 4326-4334, 2011.

[17] C. Hellriegel, J. Kirstein, and C. Bräuchle, "Tracking of single molecules as a powerful method to characterize diffusivity of organic species in mesoporous materials," New Journal of Physics, vol. 7, no. 1, pp. 1-14, 2005. UK.

[18] T. Schmidt, G. J. Schutz, W. Baumgartner, H. J. Gruber, and H. Schindler, "Characterization of photophysics and mobility of single molecules in a fluid lipid-membrane," Journal of Physical Chemistry, vol. 99, no. 49, pp. 17662-17668, 1995. Times Cited: 184 Schmidt, t schutz, gj baumgartner, w gruber, hj schindler, h.

[19] A. Kusumi, Y. Sako, and M. Yamamoto, "Confined lateral diffusion of membranereceptors as studied by single-particle tracking (nanovid microscopy) - effects of calciuminduced differentiation in cultured epithelial-cells," Biophysical Journal, vol. 65, no. 5, pp. 2021-2040, 1993. Times Cited: 382 Kusumi, a sako, y yamamoto, m.

[20] C. M. Anderson, G. N. Georgiou, I. E. G. Morrison, G. V. W. Stevenson, and R. J. Cherry, "Tracking of cell-surface receptors by fluorescence digital imaging microscopy using a charge-coupled device camera - low-density-lipoprotein and influenza-virus receptor mobility at 4-degrees-c," Journal of Cell Science, vol. 101, pp. 415-425, 1992. Part 2. 
[21] M. J. Saxton and K. Jacobson, "Single-particle tracking: Applications to membrane dynamics," Annual Review of Biophysics and Biomolecular Structure, vol. 26, pp. 373399, 1997.

[22] G. J. Schütz, H. Schindler, and T. Schmidt, "Single-molecule microscopy on model membranes reveals anomalous diffusion," Biophysical Journal, vol. 73, no. 2, pp. 1073-1080, 1997.

[23] J. Rudnick and G. Gaspari, "The shapes of random-walks," Science, vol. 237, no. 4813, pp. 384-389, 1987. ISI Document Delivery No.: J2774 Times Cited: 142 Cited Reference Count: 37 Rudnick, j gaspari, g Amer assoc advancement science Washington.

[24] C. A. Werley and W. E. Moerner, "Single-molecule nanoprobes explore defects in spingrown crystals," Journal of Physical Chemistry B, vol. 110, no. 38, pp. 18939-18944, 2006. ISI Document Delivery No.: 086DX Times Cited: 14 Cited Reference Count: 34 Werley, Christopher A. Moerner, W. E. Festschrift in honor of the 65th Birthday of Robert J Silbey Jun 24-25, 2005 Cambridge, MA Amer chemical soc Washington.

[25] B. Stempfle, M. Dill, M. Winterhalder, K. Müllen, and D. Wöll, "Single molecule diffusion and its heterogeneity during the bulk radical polymerization of styrene and methyl methacrylate," Polymer Chemistry, vol. DOI: 10.1039/C2PY20268A, 2012.

[26] D. Wöll, C. Kölbl, B. Stempfle, and A. Karrenbauer, "Novel method for automatic single molecule tracking of blinking molecules at low intensities," in preparation.

[27] H. Qian, M. P. Sheetz, and E. L. Elson, "Single-particle tracking - analysis of diffusion and flow in 2-dimensional systems," Biophysical Journal, vol. 60, no. 4, pp. 910-921, 1991.

[28] J. Schuster, F. Cichos, and C. von Borczyskowski, "Blinking of single molecules in various environments," Optics and Spectroscopy, vol. 98, no. 5, pp. 712-717, 2005. Times Cited: 29 Schuster, J Cichos, F von Borczyskowski, C.

[29] F. Köhn, J. Hofkens, R. Gronheid, M. Van der Auweraer, and F. C. De Schryver, "Parameters influencing the on- and off-times in the fluorescence intensity traces of single cyanine dye molecules," Journal of Physical Chemistry A, vol. 106, no. 19, pp. 48084814, 2002. Times Cited: 65 Kohn, F Hofkens, J Gronheid, R Van der Auweraer, M De Schryver, FC.

[30] T. Ha, T. Enderle, D. S. Chemla, P. R. Selvin, and S. Weiss, "Quantum jumps of single molecules at room temperature," Chemical Physics Letters, vol. 271, no. 1-3, pp. 1-5, 1997. Times Cited: 126 Ha, T Enderle, T Chemla, DS Selvin, PR Weiss, S.

[31] J. N. Clifford, T. D. M. Bell, P. Tinnefeld, M. Heilemann, S. M. Melnikov, J. Hotta, M. Sliwa, P. Dedecker, M. Sauer, J. Hofkens, and E. K. L. Yeow, "Fluorescence of single molecules in polymer films: Sensitivity of blinking to local environment," Journal of Physical Chemistry B, vol. 111, no. 25, pp. 6987-6991, 2007. 


\section{Appendix}

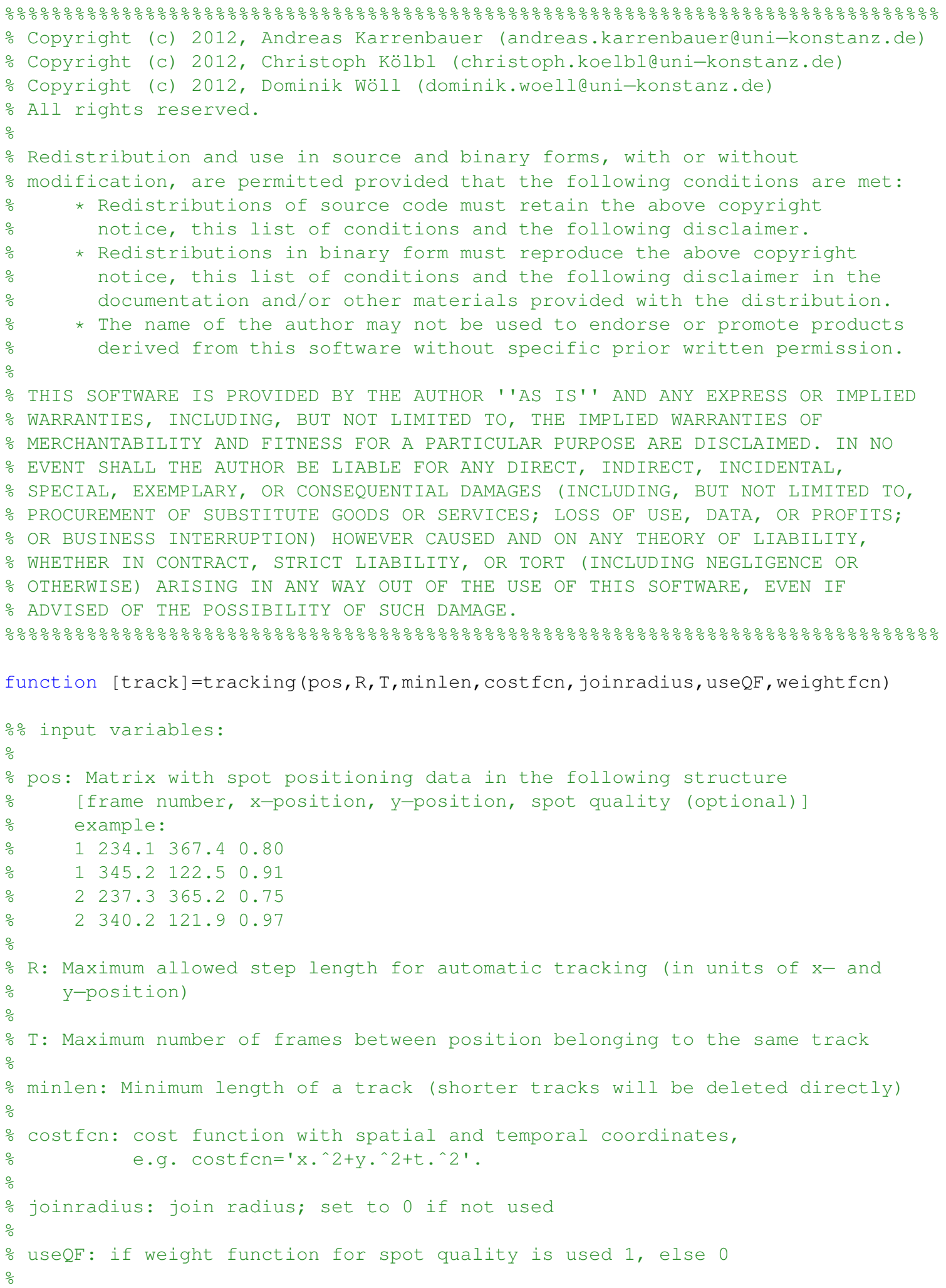




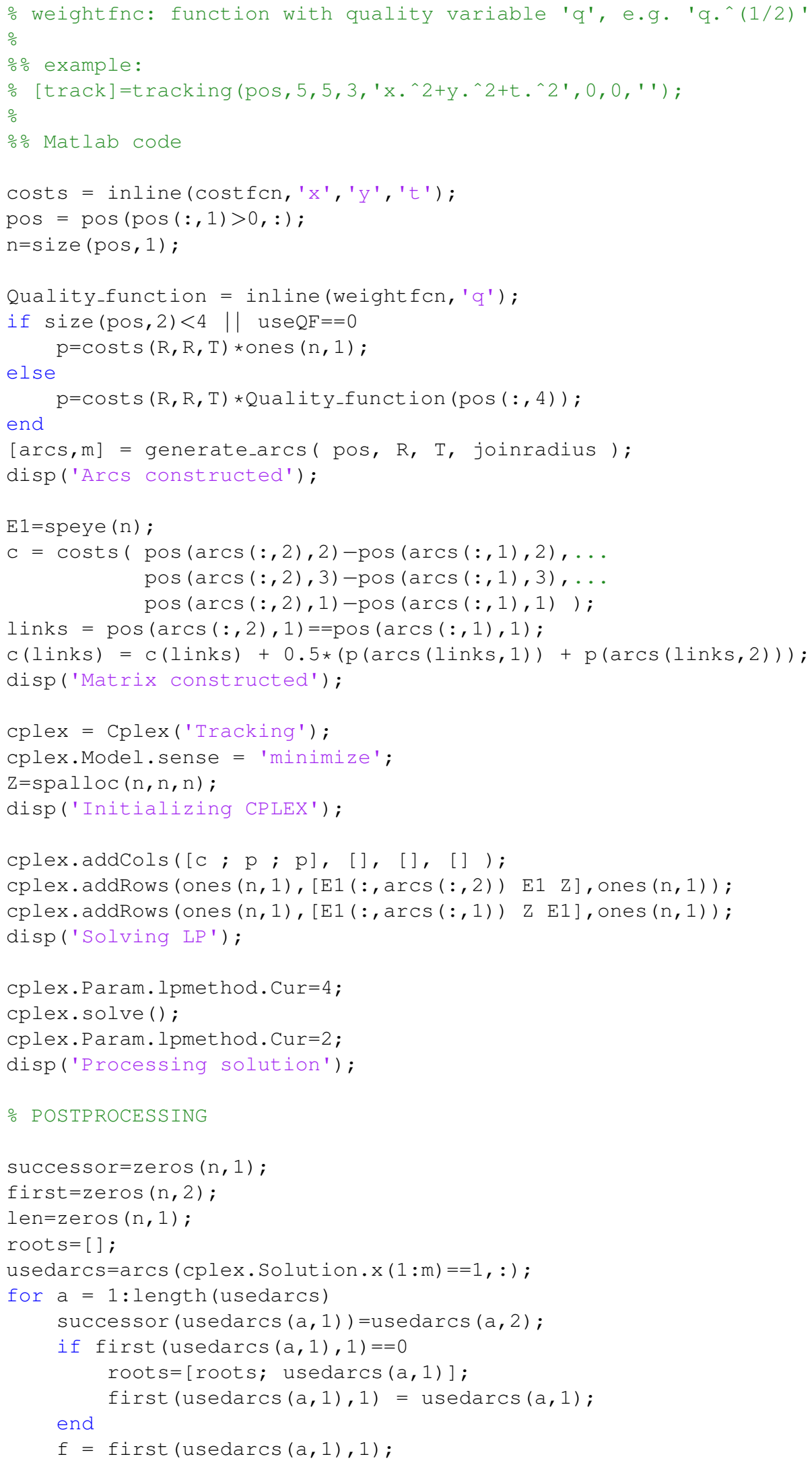









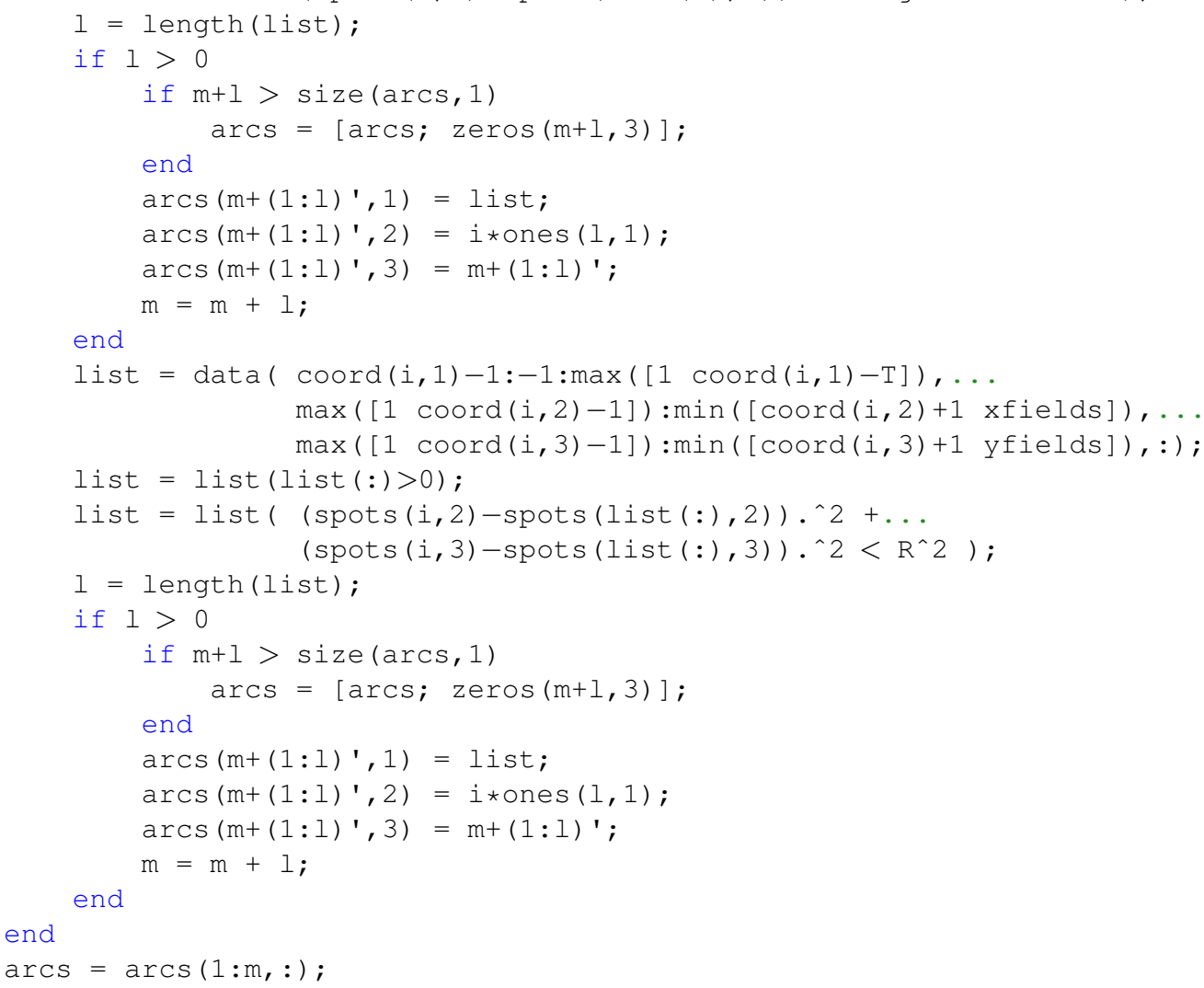

